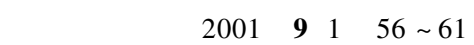

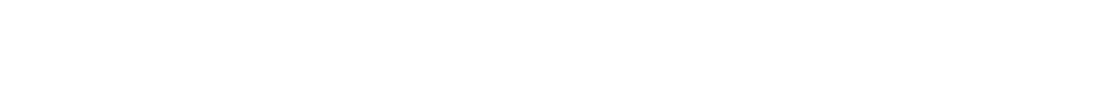

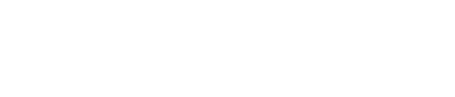

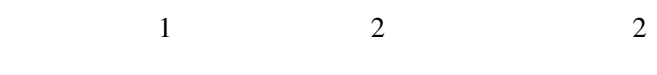

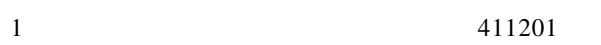

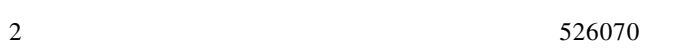

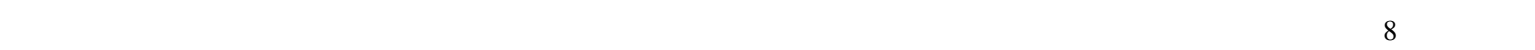

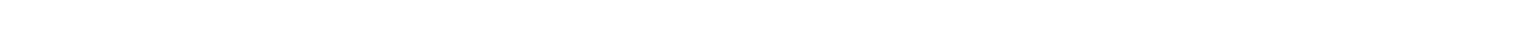



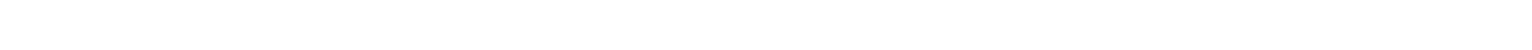

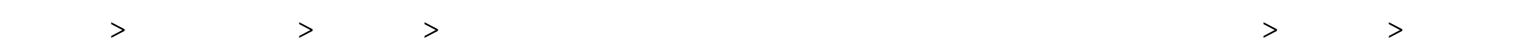

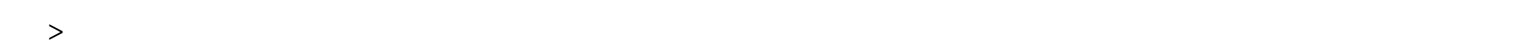

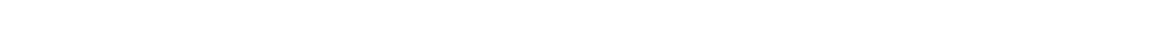

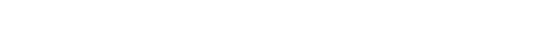

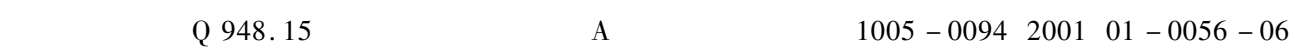

\section{Study on the species diversity of interlayer plants of Dinghushan Bio- sphere Reserve}

YI Su ${ }^{1} \mathbf{f}+\mathrm{HUANG}$ Zhong-Liang ${ }^{2} \mathbf{f}-0 \mathrm{UYANG}$ Xue-Jun ${ }^{2}$

1 Department of Biology $\mathbf{f - X i a n g t a n ~ N o r m a l ~ C o l l e g e f - X i a n g t a n f - H u n a n} 411201$

2 The management department of Dinghushan Biosphere Reservef-Zhaoqing $\mathbf{f} \neg$ Guangdong 526070

Abstract $£$-The species diversity of interlayer plants and their distribution in Dinghushan Biosphere Reserve were studied. The results showed that the species diversity of interlayer plants of Dinghushan was rich. The species diversity of lianas decreased with forest succession while epiphyte showed a contrasting trend. This suggests that the diversity indices of lianas might be employed to measure forest succession. The individual numberfspecies number and Shannon-Wiener index of lianas in ravine rain forest and riverbank forest were richer than that of other forests. These indices were the lowest in the most closed forestf`monsoon evergreen broad-leaved forest. This indicates that both humidity and illumination were important factors affecting the growth and distribution of lianas.

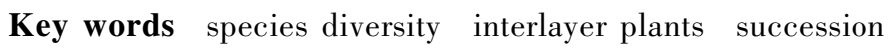

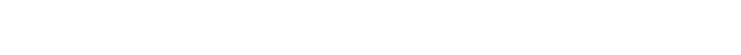

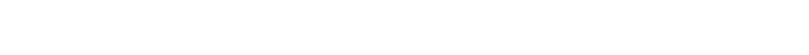

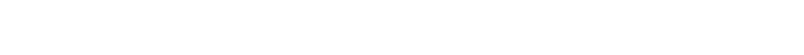

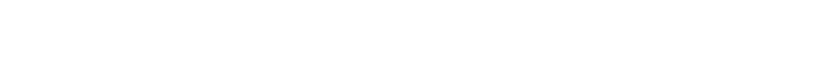

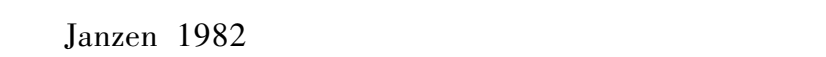

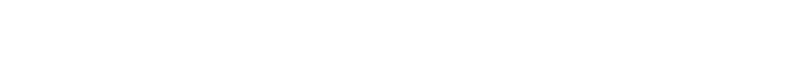

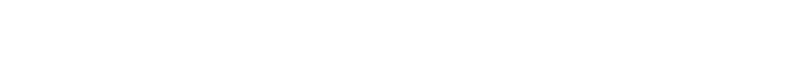

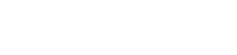

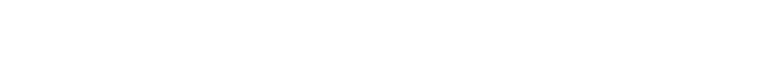

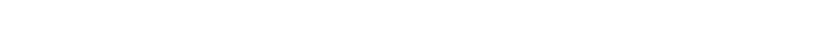

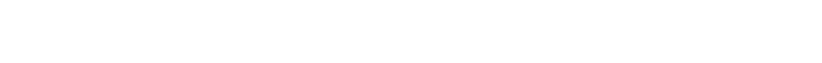

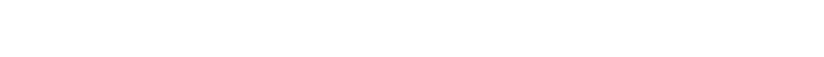

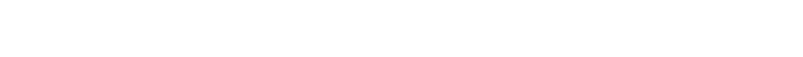

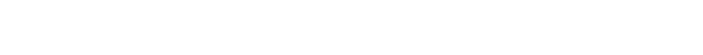




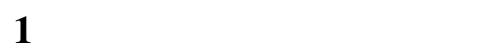

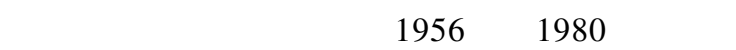

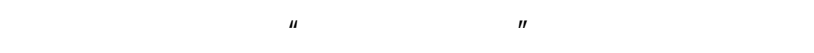

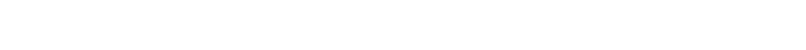
$86 \mathrm{kmfz3} 3^{\circ} 09^{\prime} 21^{\prime \prime} \sim 23^{\circ} 11^{\prime} 30^{\prime \prime} \mathrm{Nf}-12^{\circ} 30^{\prime} 39^{\prime \prime} \sim 112^{\circ}$

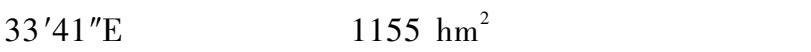

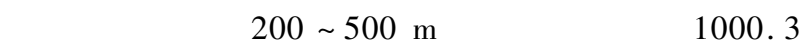



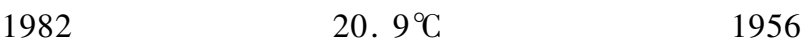

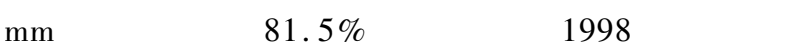







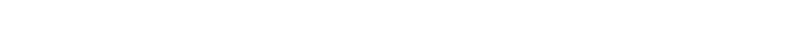

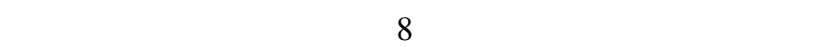

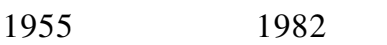

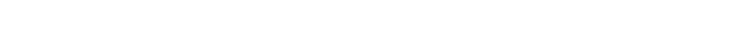

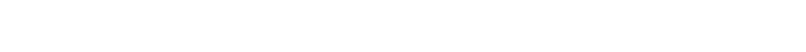

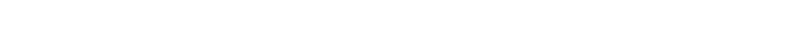

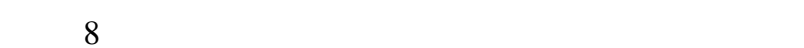

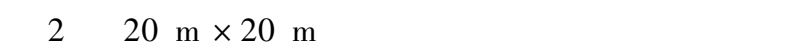

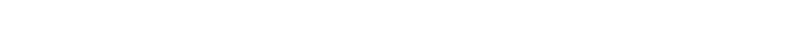

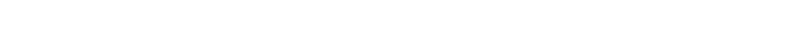



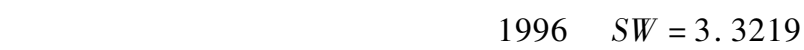
f" $\lg N-1 / N \sum n_{i} \lg n_{i} £ € £ \hat{E} 1 / \ddot{\varpi} S W$ îa Shannon-Wiener

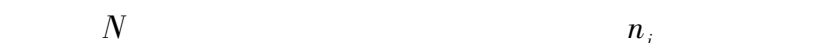
C̣̈uÚ $i$ ÖÖß̈, öl åẾy

\section{1/âṇ̂̂̈. Ö ö}


Îo

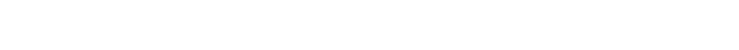

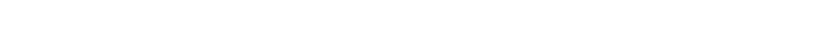

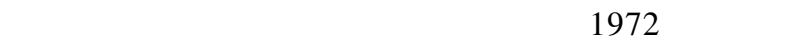

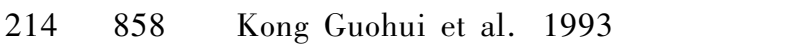

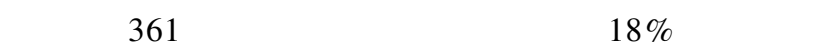

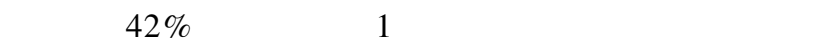

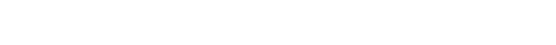

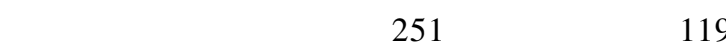

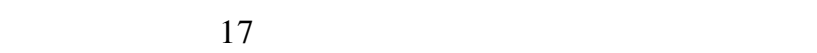

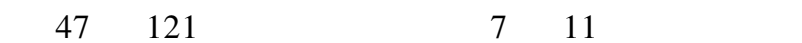

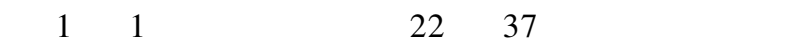

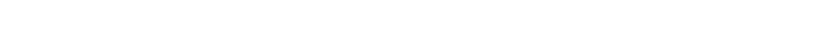
$\mu$ ã $\mathrm{f}$

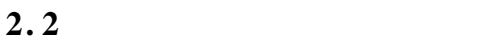

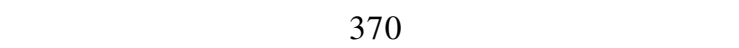

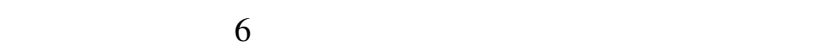

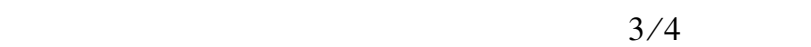

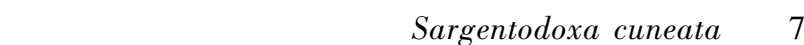

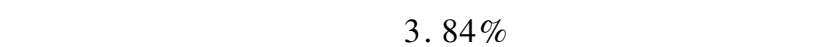

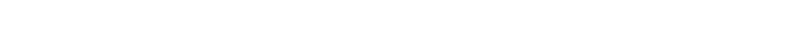

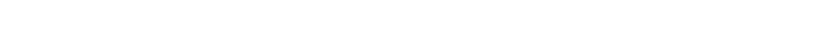

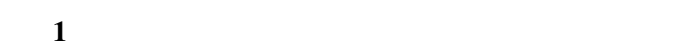

Table 1 The contribution of interlayer plants to the species pool of vascular plants of the reserve

\begin{tabular}{|c|c|c|c|c|c|c|c|c|c|c|c|c|}
\hline & \multicolumn{3}{|c|}{$\begin{array}{l}\text { P§ÀàÖ Îï } \\
\text { Fern }\end{array}$} & \multicolumn{3}{|c|}{$\begin{array}{c}\text { Âãxóö îï } \\
\text { Gymnosperm }\end{array}$} & \multicolumn{3}{|c|}{$\begin{array}{c}\text { झ\XÖÖ îï } \\
\text { Angiosperm }\end{array}$} & \multicolumn{3}{|c|}{$\begin{array}{l}\times \ddot{H} / \mathbb{A E} \\
\text { Total }\end{array}$} \\
\hline & $\begin{array}{c}¿ \mathscr{E} \\
\text { Family }\end{array}$ & $\begin{array}{c}\text { Êô } \\
\text { Genus }\end{array}$ & $\begin{array}{c}\text { ÖÖ } \\
\text { Species }\end{array}$ & $\begin{array}{c}¿ Æ E \\
\text { Family }\end{array}$ & $\begin{array}{c}\text { Ê̂̂ } \\
\text { Genus }\end{array}$ & $\begin{array}{c}\text { ÖÖ } \\
\text { Species }\end{array}$ & $\begin{array}{c}¿ \text { E } \\
\text { Family }\end{array}$ & $\begin{array}{c}\text { Êô } \\
\text { Genus }\end{array}$ & $\begin{array}{c}\text { Ö } \\
\text { Species }\end{array}$ & $\begin{array}{c}¿ Æ E \\
\text { Family }\end{array}$ & $\begin{array}{c}\text { Ê̂̂ } \\
\text { Genus }\end{array}$ & $\begin{array}{c}\text { Ö̈ } \\
\text { Species }\end{array}$ \\
\hline $\begin{array}{l}\text { Î } \rightarrow \text { ÜÖÎï } \\
\text { Vascular plants }\end{array}$ & 38 & 75 & 144 & 2 & 2 & 4 & 174 & 781 & 1824 & 214 & 858 & 1972 \\
\hline 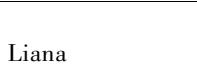 & 2 & 2 & 4 & 1 & 1 & 2 & 54 & 132 & 245 & 57 & 135 & 251 \\
\hline $\begin{array}{l}\text { 1/ÄÉÉ Ö̊̂ï } \\
\text { Parasitic plants }\end{array}$ & & & & & & & 6 & 11 & 17 & 6 & 11 & 17 \\
\hline $\begin{array}{l}2 \tilde{a}^{1} / \hat{A} \text { Ö Îï } \\
\text { Interlayer plants }\end{array}$ & 22 & 37 & 62 & 1 & 1 & 2 & 66 & 167 & 306 & 89 & 205 & 361 \\
\hline
\end{tabular}






Table 2 Species and individuals in eight natural vegetation types in Dinghushan

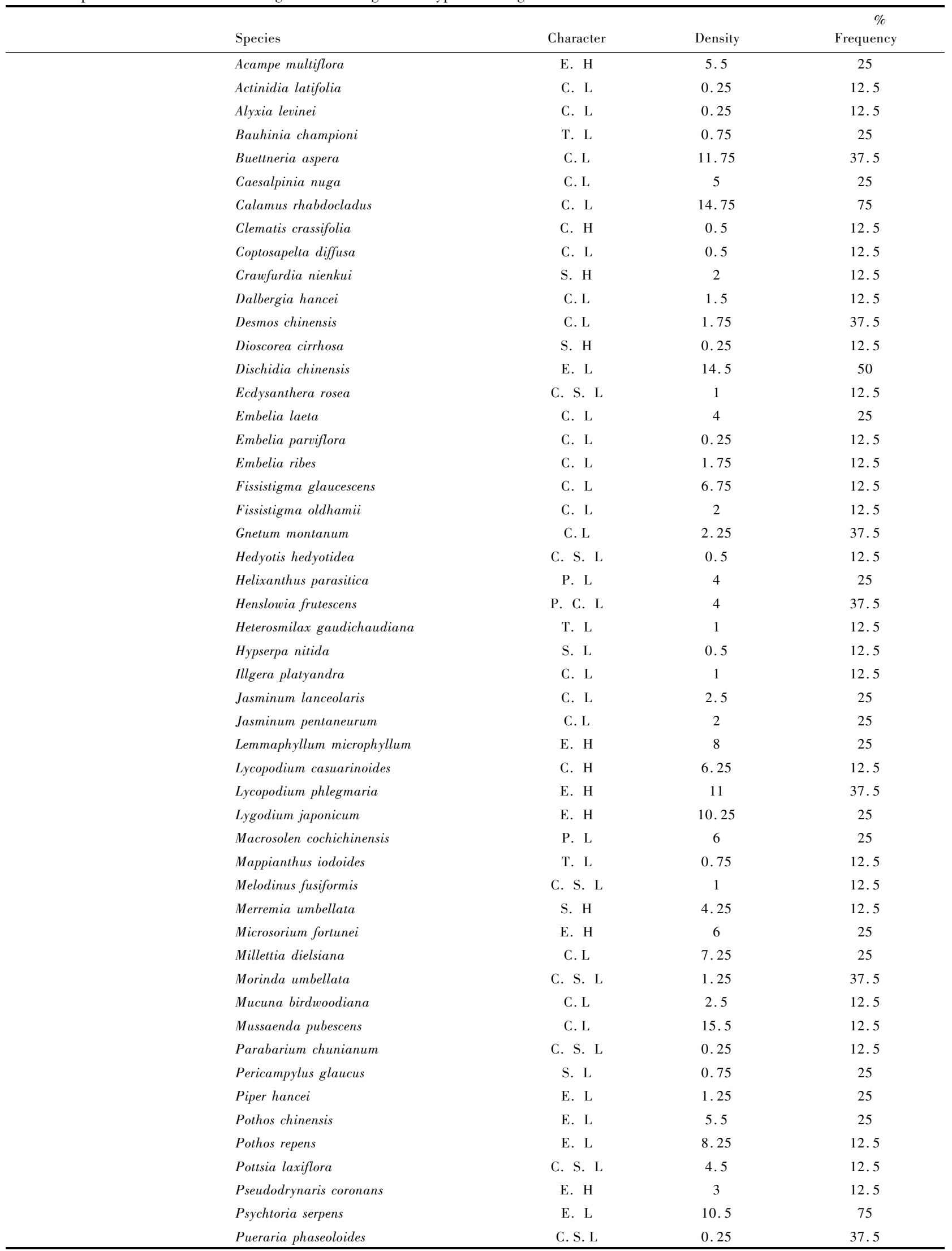




\begin{tabular}{|c|c|c|c|c|}
\hline & ÖÖÀà Species & $\begin{array}{c}\text { ĐÔx } \\
\text { Character }\end{array}$ & $\begin{array}{c}\text { qàqी̇̇ } \\
\text { Density }\end{array}$ & 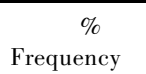 \\
\hline$\hat{E}^{\prime \prime} \times \hat{O}^{2}$ & Rhaphidophora hongkongensis & E. L & 3 & 25 \\
\hline ồ Ònì Ù & Rourea microphylla & C. L & 4 & 75 \\
\hline 'óÒnồ Òni ù & Rourea santaloides & C. L & 0.25 & 12.5 \\
\hline Ė, Ầ İ & Sargeretia theezans & C. L & 0.25 & 12.5 \\
\hline Ý̃̃ ÝÖ & Smilax chinensis & T. L & 11.75 & 25 \\
\hline Í Áübüß & Smilax glabra & T. L & 0.25 & 12.5 \\
\hline 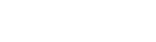 & Smilax lanceaefolia & T. L & 0.75 & 12.5 \\
\hline Ơ Ä3/4ї & Stautonia chinensis & C. $\mathrm{L}$ & 0.5 & 12.5 \\
\hline · à»pó/E & Stephania longa & S. L & 0.25 & 12.5 \\
\hline »a 1/ÄÉÉú & Taxillus chinensis & P. L & 3 & 12.5 \\
\hline শ̈ ỐÅ̊̀ & Uvaria micorcarpa & C. L & 14.75 & 37.5 \\
\hline${ }^{3}$ áoㅌㅌㅁ $\hat{u}$ & Ventilago leiocarpa & C. L & 0.25 & 12.5 \\
\hline
\end{tabular}

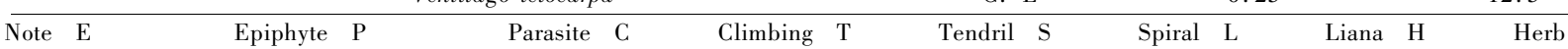



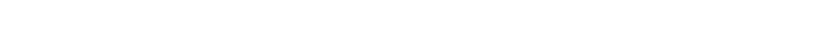

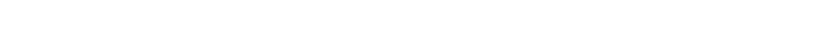

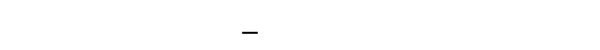

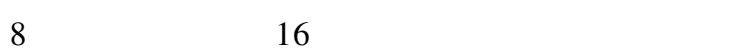
67 Ö日̈" 1 1/

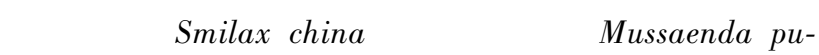

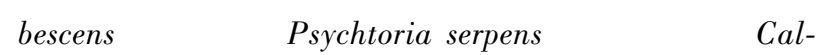

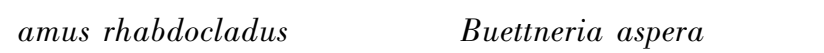

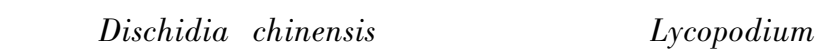

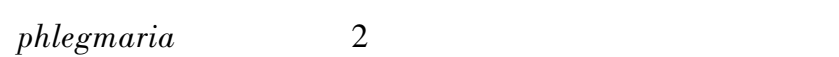

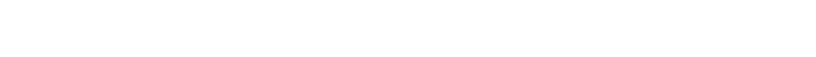

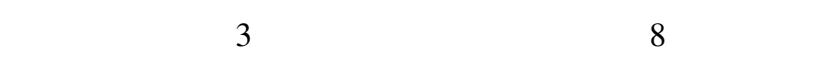

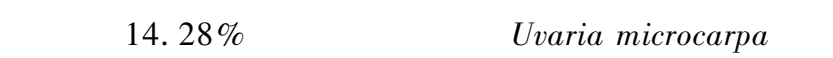

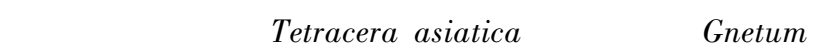

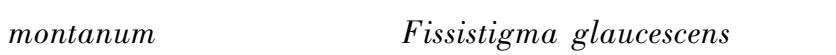

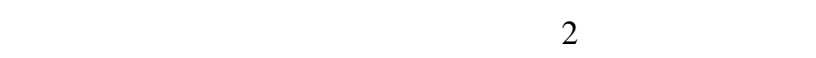

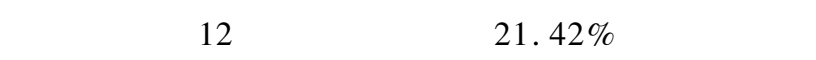
1 Ö̈Ö $\Perp$ Àà

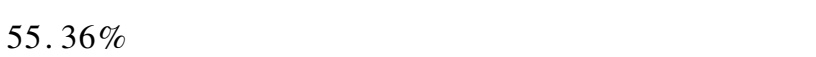

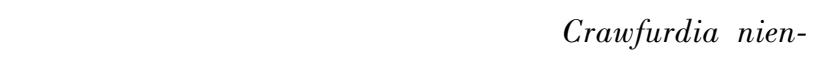

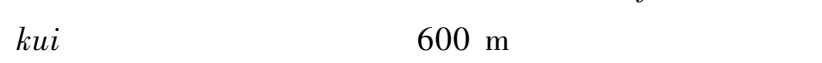
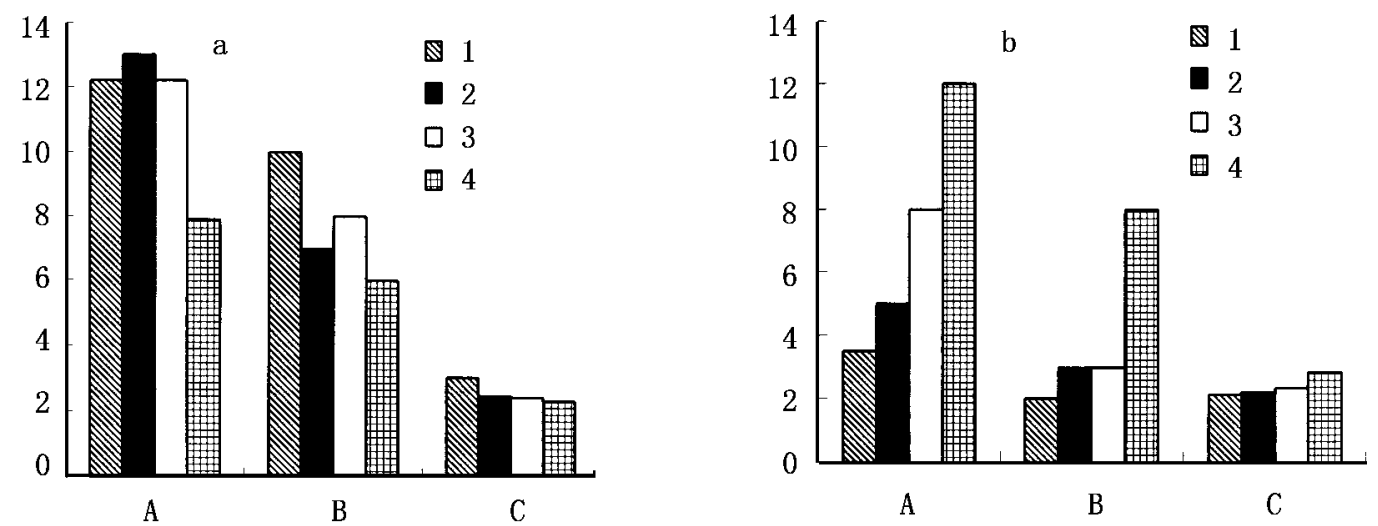

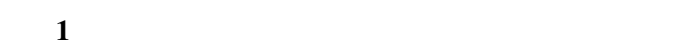

Fig. 1 Comparison of diversity along a forest succession gradient in Dinghushan

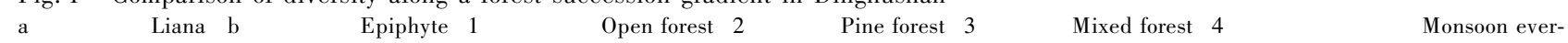

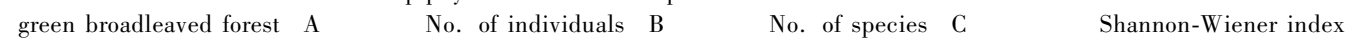




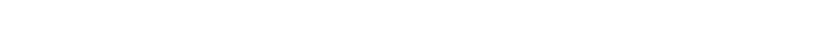



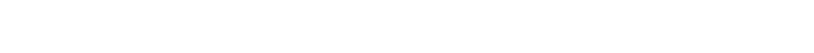

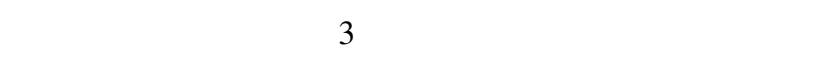

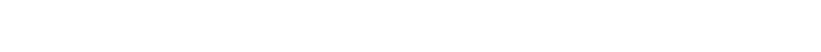

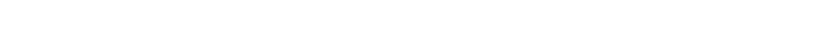

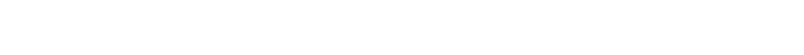

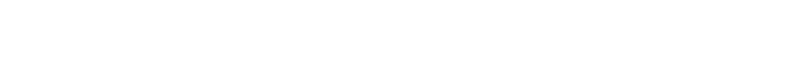

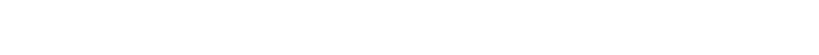

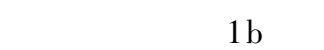

2.42 ã̃1/äÖÎ̈

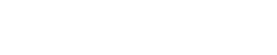

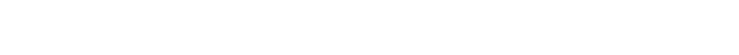

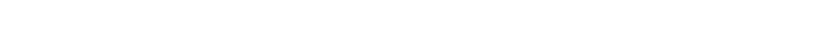

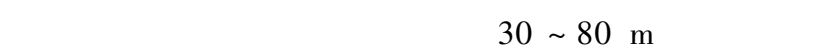

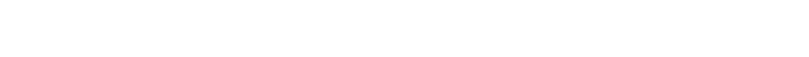

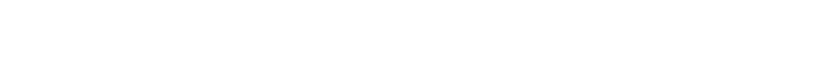

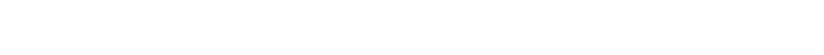

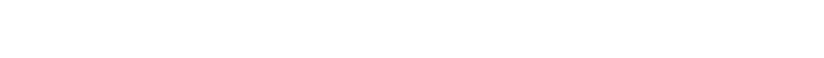
Î a Êa

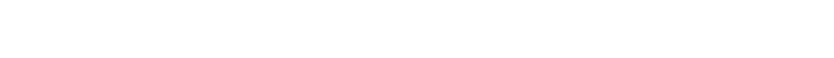

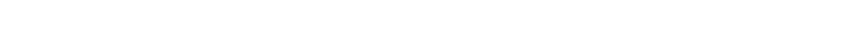

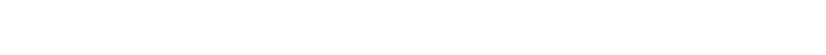



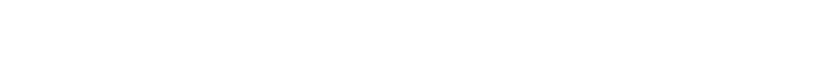

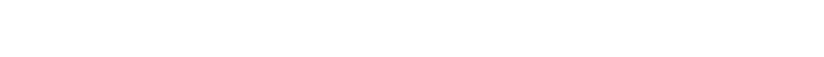

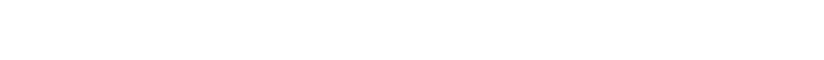

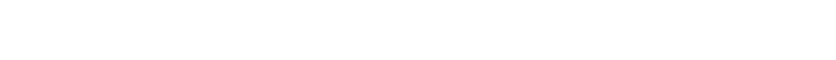

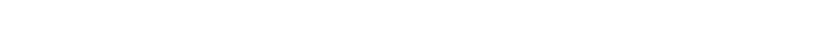

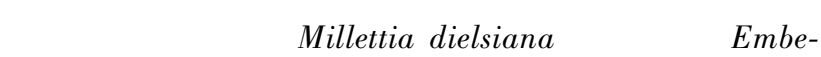

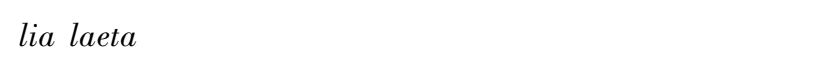

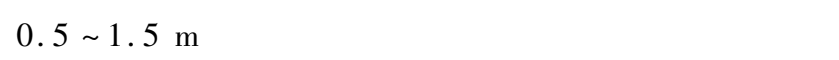

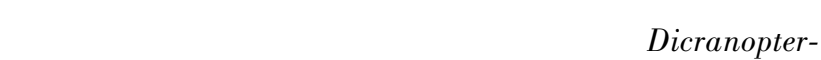

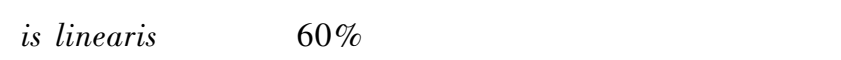

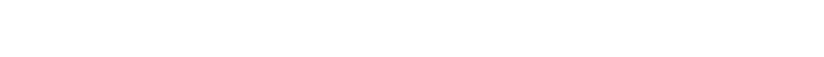

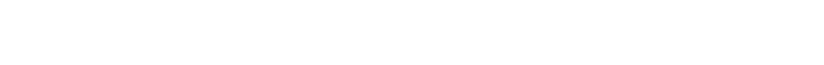

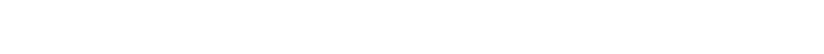

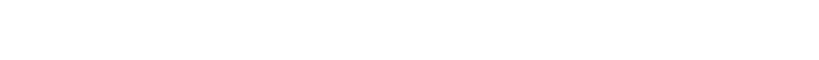

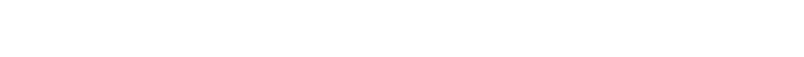

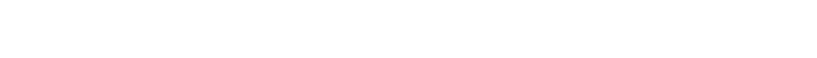

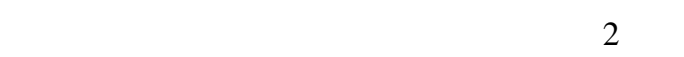

\section{3 ì ÖÂÛ}

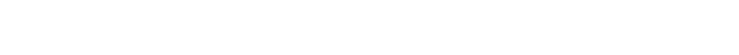

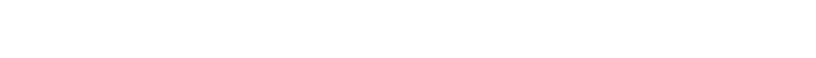



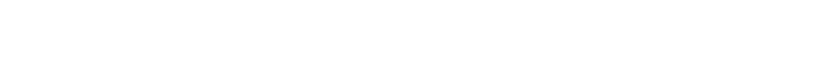

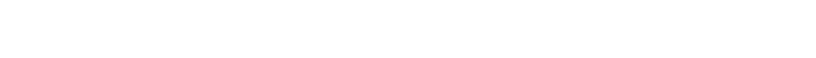
qî $\mu$ Ä, têi $\mathrm{f}$

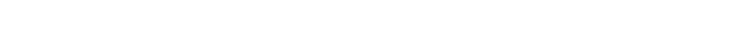

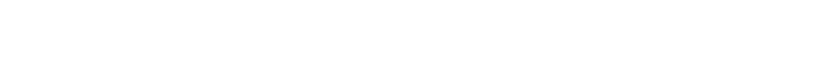

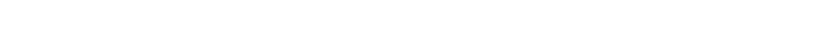

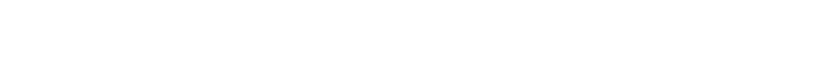

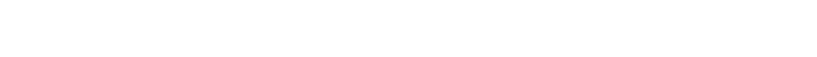

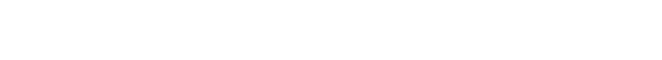

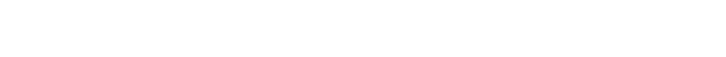

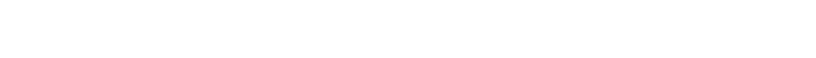
Ë山Ã



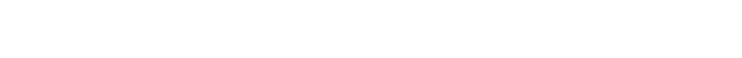

$2 \hat{\imath} \iota^{1} \hat{h} \mathrm{~A} I \ddot{I} x$

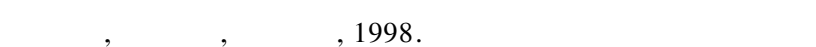

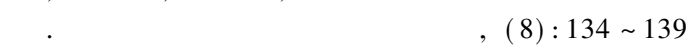

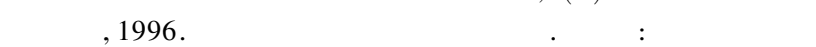

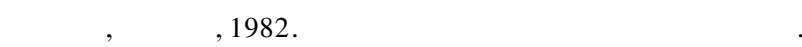

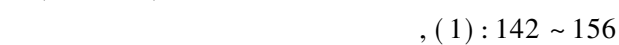

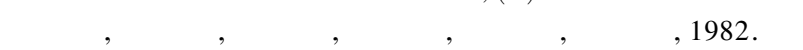

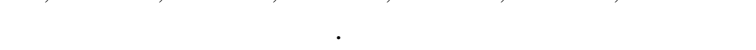

$3 / \mathfrak{d},(1): 77 \sim 141$

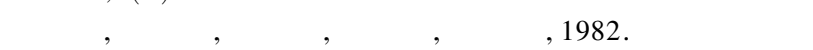

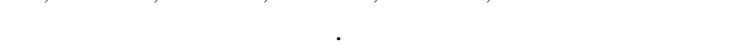
$3 / 2,(1): 1 \sim 10$

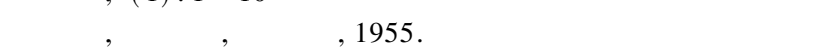

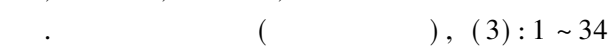

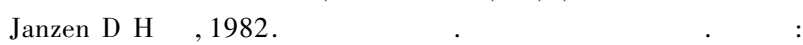

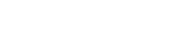

Kong G H, Liang C, Wu H M and Huang Z L, 1993. Dinghushan Biosphere Reserve ${ }^{a}$ Ecological Research History and Perspective. Beijing: Science Press 